\title{
Überdiagnostik mit Bildgebung bei Rückenschmerzen
}

\section{Qualitätssicherung mittels GKV-Routinedaten}

\author{
R. Linder ${ }^{1 *}$, D. Horenkamp-Sonntag ${ }^{1 *}$, S. Engel ${ }^{1}$, U. Schneider ${ }^{1}$, F. Verheyen ${ }^{1}$
}

\section{Zusammenfassung}

Hintergrund | Bei akuten nicht-spezifischen Rückenschmerzen sind die Beschwerden üblicherweise selbst begrenzend. Nur bei klinischem Verdacht auf einen gefährlichen Verlauf sollte gemäß Nationaler Versorgungsleitlinie Kreuzschmerz sofort eine bildgebende Diagnostik erfolgen. Ansonsten gilt es, mindestens 6 Wochen abzuwarten.

Patienten und Methodik | Mit Routinedaten der gesetzlichen Techniker Krankenkasse wurde analysiert, bei wie vielen Versicherten, die erstmalig akute Rückenschmerzen hatten und bei denen kein Hinweis auf einen gefährlichen Verlauf vorlag, eine nicht indizierte Bildgebungsdiagnostik erfolgte.

Ergebnisse | Bei ca. 10\% der Fälle erfolgte nach Diagnosestellung eine radiologische Bildge- bungsdiagnostik. Wenn eine Bildgebung durchgeführt wurde, fand sie in einem Drittel der Fälle zu früh statt oder war gänzlich unnötig. Da es sich methodisch um eine sehr konservative Schätzung handelt, steht zu vermuten, dass in der Versorgungsrealität das Ausmaß der Überdiagnostik größer ist.

Folgerungen | Bei jedem Dritten, der wegen erstmalig aufgetretener akuter nicht-spezifischer Rückenschmerzen eine radiologische Diagnostik erhält, wird die sechswöchige Wartezeit nicht eingehalten. Hochgerechnet auf die gesetzliche Krankenversicherung sind jährlich 48957 Versicherte betroffen. Überdiagnostik ist nicht nur ökonomisch problematisch, sondern hinsichtlich Patientenorientierung und -sicherheit kann sie dem Patienten auch erheblichen Schaden zufügen - entweder durch die Diagnostik selbst oder durch die Diagnostik ausgelöste Therapieformen.

\section{Einleitung}

\section{Aktueller Stand}

Die gesundheitspolitische Diskussion der letzten Zeit ist zunehmend geprägt von dem Bedarf nach Qualitätssicherung und Transparenz. Dahinter steht der Wunsch nach Verbesserungen in der Patientenversorgung, neuen Modellen zur intelligenten Steuerung über qualitätsbasierte Ressourcenallokationen bis hin zu Ansätzen in Richtung Pay for Performance oder Contracting for Outcomes. Augenfälligstes Zeichen dieses zunehmend qualitätsorientierten Denkens ist das Gesetz zur Weiterentwicklung der Finanzstruktur und der Qualität in der Gesetzlichen Krankenversicherung (GKV-FQWG). In diesem Gesetz wird die Einrichtung eines eigenständigen Instituts zur Qualitätssicherung und Transparenz im Gesundheitswesen (IQTiG) angekündigt.

Auch von den Leistungserbringern wird ein konstruktiver Wettbewerb um gute Qualität in wachsendem Maße als Chance verstanden. Den Krankenkassen als Kostenträgern erlaubt die Qualitätsorientierung, etwa über selektivvertragliche Regelungen, den Versicherten Versorgungsangebote zu machen, welche den größtmögli- chen medizinischen Nutzen bei gleichzeitiger Beachtung des Wirtschaftlichkeitsgebots gemäß $\S 12$ SGB V entfalten. Damit wird eine optimale Ressourcenallokation angestrebt. Mit der Qualitätsorientierung eröffnen sich neue Perspektiven für einen konstruktiven Service-basierten Wettbewerb.

In der Qualitätssicherung wurde lange Zeit „,vom Ende her gedacht" und damit neben Struktur-, Prozess- und Indikationsqualität [1] vor allem die Ergebnisqualität analysiert. In den Abrechnungsdaten des gesetzlichen Krankenversicherung (GKV) fehlen häufig die anamnestischen, klinischen und apparativen Detailbefunde. Daher stößt eine routinedatengestützte Untersuchung von Ergebnisqualität mit Blick auf den einzelnen Versicherten früh an Grenzen. Nach einer gewissen Ernüchterung bei der Messung der Ergebnisqualität mit Surrogatparametern [2] gewinnt aktuell die Prozessqualität an Bedeutung [3], die sich dank einer nahezu kompletten (weil abrechnungsrelevanten) Leistungshistorie in den Routinedaten hervorragend abbildet und mit den jeweiligen Leitlinienempfehlungen abgeglichen werden kann.

Dem Sachverständigenrat für die Konzertierte Aktion im Gesundheitswesen (SVRKAiG) von
Institute

1 Wissenschaftliches Institut der TK für Nutzen und Effizienz im Gesundheitswesen, Hamburg

* geteilte Erstautorenschaft

\section{Korrespondenz}

Prof. Dr. med. habil. Roland Linder Wissenschaftliches Institut der TK für Nutzen und Effizienz im Gesundheitswesen Habichtsstraße 30 22305 Hamburg Tel.: 040 / $6909-2338$ Fax.: 040 / 69 09-2307 prof.dr.roland.linder@ wineg.de 
2000/2001 zufolge lassen sich Qualitätsmängel drei Kategorien zuordnen [4]:

- Überversorgung

- Unterversorgung

- Fehlversorgung

Vor dem Hintergrund des Primats „Primum non nocere“ wird in den letzten Jahren vermehrt über Fehl- und Überversorgung nachgedacht sowie darüber, wie Patienten vor überflüssigen oder sogar schädlichen Untersuchungen und Behandlungen bewahrt werden können. Maßgeblich angeregt durch die US-amerikanische Initiative „Choosing Wisely“ wurde eine öffentliche Diskussion zum Thema Patientensicherheit angestoßen, die sich in Deutschland nur langsam entwickelt. Zuletzt wurde sie aber v.a. durch die Initiative der Deutschen Gesellschaft für Allgemeinmedizin und Familienmedizin (DEGAM) mit der Entwicklung einer entsprechenden Leitlinie „Schutz vor Überversorgung - gemeinsam entscheiden“" [5] belebt.

In diesem Zusammenhang sind wir der Frage nachgegangen, welchen Beitrag Krankenkassendaten leisten können und in welchen Handlungsfeldern die Diskussion durch eine Auswertung von GKV-Routinedaten bereichert werden kann. Einer der von Choosing Wisely [6] wie auch der Nationalen Versorgungsleitlinie Kreuzschmerz [7] inspirierten Themenbereiche, der sich ebenso in einer breit angelegten Recherche australischer Autoren zum Thema Überversorgung prominent abbildet [8] und auch die Top-5-Liste der Schweizer Initiative „Smarter Medicine“ anführt [9], ist eine potenzielle Überdiagnostik mit radiologischer Bildgebung bei Rückenschmerzen, für deren Einschätzung GKV-Routinedaten grundsätzlich geeignet sind.

\section{Überversorgung}

Wird von Überversorgung bei Patienten mit Rückenschmerz gesprochen, so lassen sich therapeutische Aspekte - etwa die Überprüfung der Indikationsstellung im Rahmen eines Zweitmeinungsverfahrens - von diagnostischen Aspekten, der sogenannten Überdiagnostik, unterscheiden. Hierunter versteht man, dass eine bildgebende Diagnostik zu früh und v.a. oftmals zu unkritisch erfolgt. Bereits im genannten Gutachten des SVRKAiG von 2000/2001 wurde dieser Punkt hervorgehoben. Daraus entstünde neben medizinischer und ökonomischer Überversorgung auch eine Fehlversorgung: Kranke würden ohne Notwendigkeit Röntgenstrahlen ausgesetzt und festgestellte Befunde führten häufig zu nicht indizierten invasiven Eingriffen [4].

An diesem Punkt setzt die Nationale Versorgungsleitlinie Kreuzschmerz [7] an, die als Langfassung seit 2010 speziell für Ärzte und seit 2011 auch für die Zielgruppe der Patienten in Form einer separaten Patientenleitlinie u. a. Ori- entierung zu Art und Umfang radiologischer Diagnostik gibt.

\section{Medizinische Definition}

Rückenschmerzen können verschiedenste Ursachen haben. Die Nationale Versorgungsleitlinie Kreuzschmerz [7] fokussiert auf die Versorgung von Patienten mit nicht-spezifischem Kreuzschmerz, definiert als Schmerz im Rückenbereich zwischen Rippenbogen und Gesäßfalten, ggf. mit Ausstrahlung.

- Neben somatischen Faktoren, z.B.

> Prädisposition,

> Funktionsfähigkeit

- sind auch psychische, z.B.

> Problemlösekompetenz,

- Selbstwirksamkeit und

- soziale Aspekte bei Krankheitsentstehung und -fortdauer relevant, z.B.

> soziale Netze,

- Versorgungsstatus,

$\checkmark$ Arbeitsplatz.

Kreuzschmerz kann nach Ursache, Dauer, Schweregrad und Chronifizierungsstadium klassifiziert werden. Ursächlich kann zwischen nicht-spezifischem und spezifischem Kreuzschmerz unterschieden werden. Für letzteren kann eine objektive Ursache wie eine Fraktur, Knochenmetastase oder ein Bandscheibenvorfall verantwortlich gemacht werden.

Der Kreuzschmerz kann auch nach dem zeitlichen Verlauf differenziert werden:

- akut: Schmerzepisoden kürzer als 6 Wochen

- subakut: länger als 6 Wochen

- chronisch/chronisch rezidivierend: Symptome bestehen bereits länger als 12 Wochen

Die Schmerzintensität kann während dieser Perioden variieren. Unter rezidivierendem Kreuzschmerz versteht man Schmerzepisoden im beschriebenen Rückenbereich, die nach einer symptomfreien Phase von mindestens 6 Monaten wieder auftreten.

\section{Prävalenz und Inzidenz}

Rückenschmerzen sind in der deutschen Bevölkerung weit verbreitet; Frauen sind häufiger betroffen als Männer. Bei einer repräsentativen ForsaUmfrage gaben lediglich $20 \%$ an, noch nie unter Rückenproblemen gelitten zu haben [10]. Es existieren nur wenige Studien, die spezifische Prävalenzen für akuten bzw. chronischen Kreuzschmerz ausweisen.

Rückenschmerzen gehören auch zu den häufigsten Gründen für Krankschreibungen. Die Gesamtzahl an Arbeitsunfähigkeitstagen von Pflichtmitgliedern der GKV lag im Jahr 2012 bei knapp 32 
Millionen [11]. Laut Robert Koch-Institut verursachten allein nicht-spezifische Rückenschmerzen im Jahr 2008 rund 3,6 Milliarden Euro an Kosten [12]. Rückenschmerzen haben in den Statistiken zur Arbeitsunfähigkeit, Frühberentung und medizinischen Rehabilitation seit Jahren einen maßgeblichen Anteil [7, 12, 13].

\section{Diagnostik und Therapie}

Bei akutem nicht-spezifischen Kreuzschmerz sind die Beschwerden üblicherweise selbst begrenzend. Folglich benötigen die meisten Patienten, die sich erstmalig wegen Rückenbeschwerden in medizinische Behandlung begeben, lediglich einer Beratung und Akutversorgung. Nur wenn klinisch der Verdacht auf einen gefährlichen Verlauf besteht, sollte gemäß der Versorgungsleitlinie Kreuzschmerz eine bildgebende Diagnostik sofort veranlasst werden. Ansonsten gilt es, mindestens sechs Wochen abzuwarten ( Abb. 1). In diesem Zeitraum sind als therapeutische Optionen Bewegung und ggf. Analgetika indiziert. Alle anderen nichtmedikamentösen Therapien wie

- Akupunktur,

- Bettruhe,

- Krankengymnastik,

- Elektrotherapie oder

- Massagen

sind genauso wenig angezeigt wie beispielsweise Phytotherapeutika oder perkutan applizierbare Medikamente. Sowohl bei einer Überdiagnostik als auch einer Übertherapie besteht die Gefahr, unbeabsichtigt zu einer Chronifizierung des Krankheitsgeschehens beizutragen.

\section{Methoden}

Unser Ziel war es, das Ausmaß einer potenziellen Überdiagnostik anhand nicht indizierter radiologischer Bildgebung zu untersuchen. Unter Verwendung von GKV-Routinedaten analysierten wir, bei wie vielen Versicherten, die erstmalig von akuten nicht-spezifischen Rückenschmerzen ohne Hinweise auf einen gefährlichen Verlauf be- troffen waren, eine zu diesem Zeitpunkt nicht indizierte radiologische Bildgebung erfolgte.

Tagesgenau wurde die radiologische Bildgebungsdiagnostik mithilfe der Gebührenordnungsziffern nach EBM (einheitlicher Bewertungsmaßstab) im ambulanten Bereich oder OPS-Codierungen (Operationen- und Prozedurenschlüssel) im stationären Sektor operationalisiert. Eine detaillierte Auflistung der Leistungen ist dem OnlineSupplement zu entnehmen.

Ausgehend von ca. 8 Millionen Versicherten der Techniker Krankenkasse im Jahr 2011 wurden diejenigen in die Analysen eingeschlossen, für die ambulant oder stationär eine gesicherte Rückenschmerz-Diagnose ICD-10-GM M54 dokumentiert war. Ausgeschlossen wurden Versicherte ohne eine mindestens einjährige lückenlose TK-Versicherung vor und nach der ersten Diagnose von Rückenschmerz in 2011. Versicherte, die nach der M54-Erstdiagnose gestorben sind, wurden nicht ausgeschlossen.

Die Spezifität des Rückenschmerzes wurde anhand des Vorliegens ambulanter und / oder. stationärer Diagnosen von Rückenschmerz verursachenden Erkrankungen operationalisiert. Dazu zählen u.a.

- Bandscheibenvorfälle,

- Unfallereignisse oder

- Tumore als Ursachen für ossäre Metastasen.

ICD-Diagnosen, die als mögliche Ursachen für spezifische Rückenschmerzen berücksichtigt wurden, sind dem Online-Supplement zu entnehmen.

Um die Analyse auf inzidente Fälle mit ausschließlich nicht-spezifischen Rückenschmerzen zu beschränken, wurden nur diejenigen Versicherten herangezogen, bei denen im Jahr vor der M54-Erstdiagnose weder Rückenschmerzen noch Ursachen für spezifische Rückenschmerzen dokumentiert waren. Ebenfalls wurden Patienten ausgeschlossen, bei denen eine mögliche Ursache für Rückenschmerz bis zu 92 Tage nach der M54Erstdiagnose dokumentiert war. Die Berücksichtigung auch zeitlich nachgelagerter Hinweise auf

\begin{tabular}{|l||l||l|}
\hline \begin{tabular}{|l|l|} 
Keine Durchführung bild- \\
gebender Untersuchungen
\end{tabular} & $\begin{array}{l}\text { Einmalige bildgebende } \\
\text { Diagnostik }\end{array}$ & $\begin{array}{l}\text { Einmalige bildgebende } \\
\text { Diagnostik }\end{array}$ \\
\begin{tabular}{|c|l|} 
Nur bei Warnhinweisen \\
nach klinischem Verdacht
\end{tabular} & $\begin{array}{l}\text { Keine Besserung ausgepräg- } \\
\text { ter und aktivitätswinschrän- } \\
\text { kender Schmerzen trotz leit- } \\
\text { liniengerechter Therapie }\end{array}$ & $\begin{array}{l}\text { Bei chronischem Schmerz- } \\
(>12 \text { Wochen) trotz leit- } \\
\text { liniengerechter Therapie }\end{array}$ \\
\hline bis zu sechs Wochen & länger als sechs Wochen & länger als zwölf Wochen \\
\hline
\end{tabular}

Abb. 1 Durchführung bildgebender Diagnostik in Abhängigkeit vom zeitlichen Verlauf der Schmerzsymptomatik gemäß Nationaler Versorgungsleitlinie Kreuzschmerz [7]. 


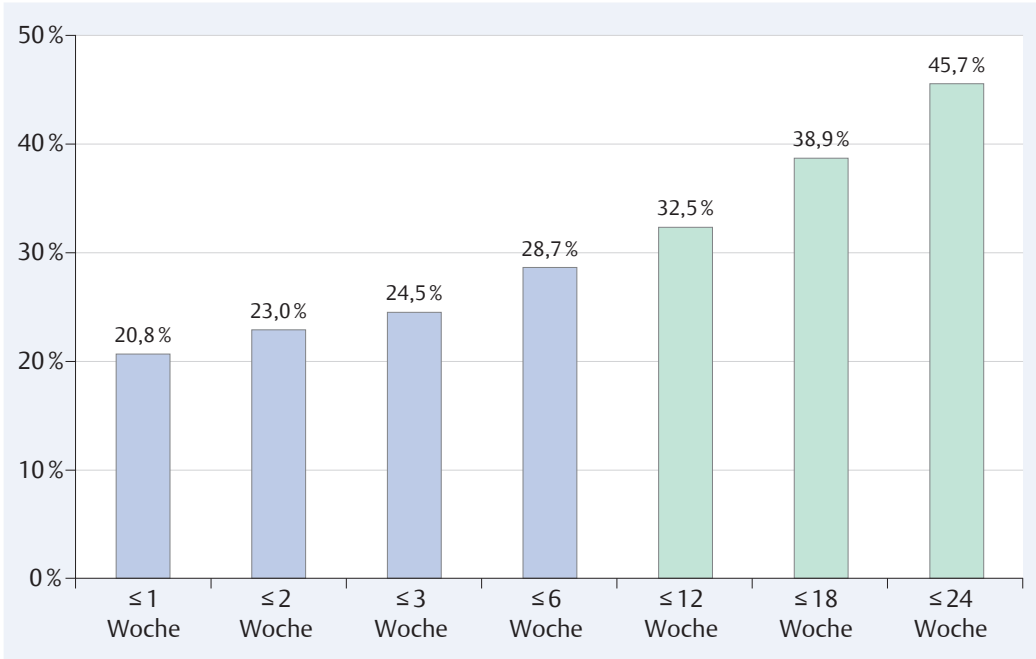

Abb. 2 Wartezeit von Erstdiagnose „Rückenschmerzen" bis zur radiologischen Bildgebung. Ordinate: Anteil Versicherter mit radiologischer Erstdiagnostik an allen Versicherten mit radiologischer Diagnostik, die ausschließlich nach Beginn der Rückenschmerzen erfolgt ist. eine Spezifität des Rückenschmerzes ist zwar konservativ, trägt jedoch solchen Konstellationen Rechnung, in denen ein bereits vorbestehender aber noch undokumentierter Hinweis auf eine spezifische Ursache bestand.

Eine besondere Schwierigkeit der Analyse von Routinedaten besteht darin, dass die Diagnosedaten in der vertragsärztlichen Versorgung ( $\$ 295$ SGB V) nicht tagesgenau, sondern nur quartalsweise dokumentiert vorliegen. Um eine zeitliche Vergleichbarkeit mit den tagesgenauen Diagnosen aus dem stationären Bereich herzustellen, wurden alle ambulanten Diagnosedaten einheitlich auf den ersten Tag des jeweiligen Quartals vordatiert. Dieses Vorgehen erlaubt es jedoch nicht, eine leitlinienkonforme Unterschreitung des 6-Wochen-Intervalls zwischen Erstdiagnose und Bildgebung zuverlässig zu bestimmen.

Als Lösung für diese methodische Limitation wurden die Analysen zunächst mit derjenigen Subpopulation an Versicherten durchgeführt, für die zusätzlich zur ambulanten Diagnose tagesgenaue Informationen zur Arbeitsunfähigkeit (AU, $\S 44$ SGB V) wegen Rückenschmerzen vorlagen. Der so ermittelte Prozentsatz an Versicherten mit Bildgebung im 6-Wochenzeitraum nach Erstdiagnose wurde anschließend zur Analyse der Gesamtpopulation in 2011 genutzt.

\section{Ergebnisse}

In den Voranalysen mit den AU-Daten hatten 2155 Versicherte im Zeitraum 2011-2012 akute nicht-spezifische Rückenschmerzen. Bei 265 Fällen war eine radiologische Bildgebung ausschließlich nach dem Datum der M54-Erstdiagnose im Leistungsverlauf dokumentiert, wobei diese in $28,7 \%$ der Fälle $(n=76)$ innerhalb der ersten 6 Wochen durchgeführt wurde ( $\bullet$ Abb. 2).
Für die Hauptanalyse der TK-Versicherten mit M54-Diagnose in 2011 galt:

- 1559446 TK-Versicherte hatten im Jahr 2011 mindestens eine gesicherte ambulante oder stationäre Rückenschmerz-Diagnose.

- 77416 (5,0\%) davon hatten vor der ersten M54-Diagnose weder Rückenschmerz verursachende Krankheiten noch Tumorerkrankungen im Krankheitsverlauf.

- Bei 23354 dieser 77416 Versicherten (30,2\%) war eine radiologische Bildgebung im Leistungsverlauf dokumentiert, bei 19482 davon ausschließlich nach der ersten ICD-M54Diagnose.

- Bei Anwendung des Prozentsatzes von 28,7\% wurde demnach bei 5591 Versicherten innerhalb der ersten sechs Wochen nach Beginn der Rückenschmerzen entgegen der Versorgungsleitlinie eine radiologische Diagnostik durchgeführt.

Viele Versicherte wurden aus der Analyse ausgeschlossen, weil sie chronischen Rückenschmerz hatten oder an Krankheiten litten, die potenziell Rückenschmerz verursachen können. Hierzu zählen

- Gelenkkrankheiten (46,4\%),

- Unfallverletzungen (44,1\%),

- Erkrankungen der Wirbelsäule exkl. M54 $(54,9 \%)$

- Erkrankungen von Muskeln, Sehnen, Bindegewebe oder Weichteilen $(48,4 \%)$,

- angeborene Fehlbildungen (17,9\%) und

- Tumorerkrankungen $(8,8 \%)$

Bei mehr als einer Million Versicherten (67,3\%) waren Rückenschmerzen als Vorerkrankung bekannt ( Abb. 3).

\section{Diskussion}

Bei der Analyse von GKV-Routinedaten ist stets zu bedenken, dass es sich um Sekundärdaten handelt, die nicht alle klinischen Informationen enthalten [14-17]. Um dieses zu kompensieren, haben wir alle Krankheitskonstellationen ausgeschlossen, die potenziell relevant für die Pathogenese von Rückenschmerzen sein können bzw. bei denen möglicherweise „red flags“ vorgelegen haben könnten. Dies kann als ein Grund für die niedrige Inzidenz von akutem nicht-spezifischem Rückenschmerz gelten.

Zum anderen wurde hinsichtlich der Kausalität bewusst ein großer zeitlicher Sicherheitsabstand berücksichtigt, um eine potenziell verzögerte Dokumentation klinisch relevanter Informationen in GKV-Routinedaten zu berücksichtigen. So ist es vorstellbar, dass Rückenschmerzen infolge von ossären Metastasen bei undokumentiertem Verdacht Auslöser für eine Tumordiagnostik bei einem Versicherten waren und die entsprechenden Diagnoseinformationen zum Tumor erst zeitlich 
versetzt in den GKV-Routinedaten dokumentiert wurden. Auch wenn hier innerhalb des 6-Wochen-Intervalls nach Erstdiagnostik eines Rückenschmerzes eine Radiologie erfolgte, wurden derartige Konstellationen bewusst von den Analysen ausgeschlossen. Insofern darf die vorliegende Operationalisierung als konservativ gelten, d.h. es ist von einer Unterschätzung des Ausmaßes der Leitlinienabweichung auszugehen.

In der Mehrzahl der Fälle (>90\%) wird auf eine nicht indizierte oder zu früh durchgeführte radiologische Bildgebungsdiagnostik verzichtet und die Leitlinie damit adäquat umgesetzt. Wenn aber eine Bildgebung erfolgt, findet sie in einem Drittel dieser Fälle zu früh statt oder ist gänzlich unnötig. Hierbei ist auffällig, dass die Bildgebung in $74,2 \%$ der Fälle innerhalb der ersten sieben Tage nach der Erstdiagnose erfolgt. Aus den vorgenannten Gründen steht jedoch zu vermuten, dass in der Versorgungsrealität die tatsächliche Zahl von Patienten mit zu früher oder gänzlich überflüssiger radiologischer Diagnostik noch höher liegt.

Die radiologischen Leistungen wurden bewusst sehr umfassend in die Analyse eingeschlossen, um möglichst keine radiologische Leistungserbringung zu übersehen. Alle potenziell in Frage kommenden EBM-Ziffern (ambulanter Sektor) und OPS-Codes (stationärer Sektor) wurden berücksichtigt. Dieses führt möglicherweise zu Unterschätzungen von konventionellen Röntgenleistungen, da diese im stationären Sektor nicht separat kodiert werden. Dafür könnten bei CT- und MRTUntersuchungen eventuell Überschätzungen vor- liegen, wenn diese im stationären Bereich wegen anderer Krankheitsindikationen zeitlich nachgelagert zur M54-Erstdiagnose durchgeführt wurden.

Eine bildgebende Diagnostik kann zum Ausschluss einer spezifischen Ursache führen und dann folgerichtig als nicht-spezifischer Rückenschmerz in den Daten verbleiben, wenn keine der möglichen Ausschlussgründe im Rahmen der ärztlichen Dokumentation kodiert werden sollte (falsch-positiver Fall). Auf der anderen Seite ist zu berücksichtigen, dass in unseren Analysen sämtliche Fälle ausgeschlossen worden sind, bei denen auch ohne Vorliegen einer red-flag-Situation geröntgt worden ist und die dann festgestellten radiologischen (Zufalls-) Befunde in diesen Fällen erst zur Dokumentation von radiologisch motivierten ICD-Ausschlussdiagnosen geführt haben (falsch-negative Fälle).

GKV-Routinedaten werden im Rahmen der (ärztlichen) Leistungserbringung dokumentiert und fallen somit zeitlich nach dem Auftreten erster (klinischer) Symptome an. Es gibt Indikationen, bei denen das Zeitfenster zwischen klinischem Krankheitsbeginn und dokumentierter ICDMorbidität gering ist (z. B. Myokardinfarkt) und Indikationen mit größerer Latenzzeit (z. B. Fußpilz).

Es wurden nur Patienten untersucht, die noch nie in ihrer Vorgeschichte Rückenschmerzen hatten. Da bei AU ein monetärer Anreiz besteht, frühzeitig einen Arzt aufzusuchen und andererseits nur diejenigen Patienten erfasst werden, die so sehr unter den erstmalig aufgetretenen Schmerzen leiden, dass sie ihrer Arbeit nicht nachgehen kön-

Abb. 3 Art und Umfang von Rückenschmerz verursachenden Diagnosen bei Versicherten mit Rückenschmerz (ICD M54). Ordinate: Anteil von Versicherten mit Rückenschmerzen verursachenden Erkrankungen.

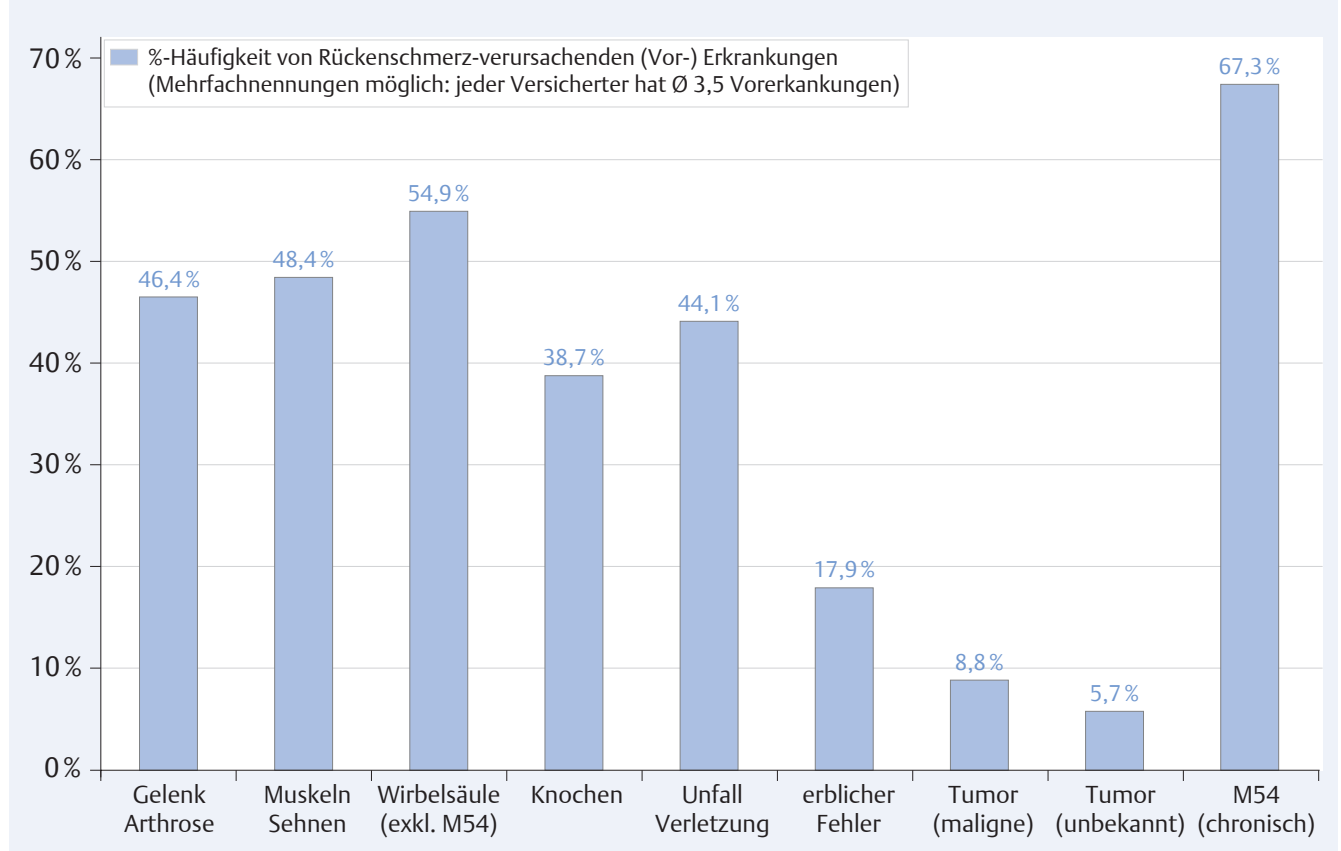


nen, gehen wir von einer methodisch korrekten Operationalisierung aus. Die AU-Subgruppe ( $n=265$ Versicherte) unterschied sich vom Gesamtkollektiv in den ambulanten und stationären TK-Daten $(\mathrm{n}=19482)$ hinsichtlich der Altersstruktur nur geringfügig (Mittelwert 45,2 vs. 45,6 Jahre), während beim Geschlecht der Männeranteil in der AU-Subgruppe etwas höher (61,5\% vs. 52,9\%) war.

\section{Ursachenanalyse - mögliche Einflussfaktoren}

Unsere Ergebnisse liefern Hinweise darauf, dass die Leitlinie zum Rückenschmerz in der Versorgungswirklichkeit nicht ausreichend Beachtung findet. Es steht zu vermuten, dass viele Faktoren einen Einfluss auf eine vorzeitige radiologische Diagnostik haben: An erster Stelle der Versicherte, der sich ggf. von wenig validen Informationsangeboten verleiten lässt, diagnostische (HighTech-) Möglichkeiten für sich in Anspruch nehmen zu wollen. Doch auch den Ärzten als Leistungserbringern mag eine besondere Bedeutung zukommen. Einerseits könnte der Hausarzt zur Defensivmedizin neigen, anderseits der Facharzt die Amortisierung seiner Praxisausstattung anstreben. Zudem scheuen womöglich beide Berufsgruppen die gegebenenfalls erforderliche, aber oft zeitintensive Überzeugungsarbeit beim Patienten, dass trotz vorhandener Schmerzsymptomatik ad hoc keine akute Diagnostik erforderlich ist. Auch kann der Patient bei vermeintlich zu restriktivem Vorgehen an die benachbarte Praxis verloren werden.

Letztlich müssen sich alle Beteiligten (einschließlich der Krankenkassen) fragen, wie Aspekte der Überversorgung thematisiert werden können, ohne dass Leistungen - in der Wahrnehmung der Versicherten - vorenthalten werden, sondern unnötige und teils kontraproduktive Ausgaben vermieden werden.

Bei der Arztgruppe des zum Radiologen überweisenden Arztes lässt sich nicht valide zwischen Haus- und Facharzt differenzieren. Es besteht weder Wissen darüber, wie sich Patientenströme auf die Haus- und Fachärzte verteilen, noch welche Besonderheiten es in der Zusammensetzung dieser Patientenströme gibt.

Zu diesen Besonderheiten zählt, dass bei der untersuchten Krankheitsindikation, den akuten Rückenschmerzen, die Akuität der Schmerzsymptomatik im Vordergrund stand. Daher dürfte bei der ersten ärztlichen Leistungsinanspruchnahme der Zeitfaktor bei der Terminvergabe (Wartezeit auf einen Facharzttermin) der dominierende Faktor hinsichtlich der Arztgruppenauswahl sein. Zudem können auch monetäre Anreize durch die sogenannte Praxisgebühr, die bis 2012 ein erst- maliges Aufsuchen des Hausarztes gefördert hat, eine Rolle gespielt haben.

Daher kann das Ergebnis unserer Analysen, dass 73,7\% aller Überweisungen zum Radiologen vom Hausarzt (hausärztlich niedergelassene Internisten, Allgemeinmediziner und praktische Ärzte) ausgestellt worden sind und der Anteil von Orthopäden, Unfallchirurgen und Chirurgen bei unter $10 \%$ liegt, in keiner Weise im Sinne irgendeiner „Schuldzuweisung“ interpretiert werden.

\section{Handlungsoptionen}

Die Ergebnisse machen deutlich, dass das Thema Überdiagnostik ein relevantes Problem darstellt, nicht nur aus ökonomischer Sicht, sondern auch im Hinblick auf die Patientenorientierung und -sicherheit. Dem Patienten kann erheblicher Schaden - sei es durch die Diagnostik selbst oder durch im Rahmen der Diagnostik ausgelöste Therapie - zugefügt werden [18-20]. Daher ist bei allen Beteiligten eine weitergehende Sensibilisierung erforderlich.

\section{Fazit und Ausblick}

Bei jedem dritten Versicherten, der wegen erstmalig aufgetretener akuter nicht-spezifischer Rückenschmerzen eine radiologische Bildgebung erhält, wird die sechswöchige Wartezeit nicht eingehalten. Ausgehend von einem TKMarktanteil von $11,4 \%$ an allen gesetzlich krankenversicherten Personen in Deutschland in 2011 sind hochgerechnet auf die GKV jährlich 48957 Versicherte betroffen. Angesichts des Krankheitsbildes mit üblicherweise selbst begrenzenden Beschwerden werden diese Menschen einer unnötigen Strahlenbelastung ausgesetzt. Zudem kann Überdiagnostik auch zu einer Chronifizierung führen und häufig nicht indizierte invasive Eingriffe zur Folge haben. Deshalb ist vor jeder radiologischen Bildgebung kritisch zu hinterfragen, inwieweit eine Indikationsstellung wirklich gegeben ist.

Hinsichtlich künftiger Weiterentwicklungen in der Qualitätssicherung stellen GKV-Routinedaten eine wertvolle Quelle dar, vorliegend gezeigt am Beispiel des Abgleichs der Versorgungswirklichkeit von Patienten mit Rückenschmerz gegen die entsprechende Leitlinienempfehlung. Weitere Untersuchungen zur Umsetzung der PRISCUSListe [21], der Nationalen Versorgungsleitlinie zur Koronaren Herzkrankheit [22, 23] oder der GBA-Richtlinie zur Stufendiagnostik [24] bei schlafbezogenen Atemstörungen unterstreichen das Potenzial der Routinedaten. 
Konsequenzen für Klinik und Praxis

- Bei akuten nicht-spezifischen Rückenschmerzen ohne klinischen Verdacht auf einen gefährlichen Verlauf sollte erst nach einer Wartezeit von sechs Wochen radiologisch untersucht werden.

- In ca. 10\% der Fälle erfolgt nach Diagnosestellung eine radiologische Bildgebung.

- Wenn eine radiologische Bildgebungsdiagnostik durchgeführt wird, findet diese in einem Drittel der Fälle zu früh oder gänzlich unnötig statt.

- Hochgerechnet auf die GKV wird jährlich bei 48957 Versicherten vor Ablauf der sechswöchigen Wartefrist eine bildgebende Diagnostik durchgeführt.

\section{Literatur}

1 Kraft R, Hersperger M, Herren D. Diagnose und Indikation als Schlüsseldimensionen der Qualität. Schweizerische Ärztezeitung 2012; 93: 1485-1489

2 Mühlhauser I, Müller H. Patientenrelevante Endpunkte und patient-reported-outcomes in klinischer Forschung und medizinischer Praxis. In: Klusen N, Fließgarten A, Nebling T, Hrsg. Informiert und selbstbestimmt. Der mündige Bürger als mündiger Patient. Baden-Baden: Nomos; 2009: 34-64

3 Schrappe M. Qualität 2030 - Die umfassende Strategie für das Gesundheitswesen. Berlin: Medizinisch Wissenschaftliche Verlagsgesellschaft; 2015

4 Sachverständigenrat für die Konzertierte Aktion im Gesundheitswesen. Gutachten 2000/2001 - Bedarfsgerechtigkeit und Wirtschaftlichkeit. Band III: Über-, Unter- und Fehlversorgung. Baden-Baden: Nomos; 2002

5 Deutsche Gesellschaft für Allgemeinmedizin und Familienmedizin. Schutz vor Überversorgung gemeinsam entscheiden. Angemeldete S3Leitlinie, Register-Nummer 053-045. http://www.awmf.org/leitlinien/detail/ anmeldung/1/II/053-045.html Letzter Zugriff am 07.03.16

6 American Academy of Family Physicians. Imaging tests for lower-back pain. http://www.choosingwisely.org/doctor-patientlists/imaging-tests-for-lower-back-pain/ Letzter Zugriff am 07.03.16

7 Bundesärztekammer, Kassenärztliche Bundesvereinigung, Arbeitsgemeinschaft der Wissenschaftlichen Medizinischen Fachgesellschaften. Nationale VersorgungsLeitlinie: Kreuzschmerz. Langfassung Version 4, 2010, zuletzt verändert: August 2013. DOI: 10.6101/AZQ/000149

8 Elshaug AG, Watt AM, Mundy L, Willis CD. Over 150 potentially low-value health care practices: an Australian study. Med J Aust 2012; 197: 556-560

9 Selby K, Cornuz ], Neuner-Jehle S et al. «Smarter Medicine»: 5 Interventionen, die in der ambulanten allgemeinen inneren Medizin vermieden werden sollten. Schweizerische Ärztezeitung 2014; 95 : 769-770

10 Techniker Krankenkasse. Deutschland hat Rücken Der TK-Rückenreport. Medienservice März 2012. http://www.bodylife.com/uploads/media/ TK-Rueckenreport.pdf Letzter Zugriff am 29.03.2016
11 Bundesministerium für Gesundheit. Arbeitsunfähigkeit: Fälle und Tage nach Diagnosen 2012, Ergebnisse der Krankheitsartenstatistik der gesetzlichen Krankenversicherung. http://bmg.bund.de/fileadmin/dateien/Downloads/Statistiken/GKV/Geschaeftsergebnisse/AU_ nach_Diagnosen_2012.pdf Letzter Zugriff am 07.03.2016

12 Raspe H. Rückenschmerzen. Gesundheitsberichterstattung des Bundes. Heft 53.

http://www.rki.de/DE/Content/Gesundheitsmonitoring/Gesundheitsberichterstattung/ GBEDownloadsT/rueckenschmerzen.pdf? blob=publicationFile

Letzter Zugriff am 07.03.2016

13 Bungard S, Hertle D, Kliner K et al. BKK Gesundheitsreport 2013. Gesundheit in Bewegung. Schwerpunkt Muskel- und Skeletterkrankungen. Berlin: BKK Bundesverband 2013.

http://www.bkk-dachverband.de/fileadmin/ publikationen/gesundheitsreport/ fruehere_gesundheitsreporte/ BKK-Gesundheitsreport_2013.pdf Letzter Zugriffam 29.03.2016

14 Arbeitsgruppe Erhebung und Nutzung von Sekundärdaten der Deutschen Gesellschaft für Sozialmedizin und Prävention und der Deutschen Gesellschaft für Epidemiologie. Gute Praxis Sekundärdatenanalyse (GPS), Leitlinien und Empfehlungen. 3. Fassung 2012, geringfügig modifiziert 2014

http://dgepi.de/fileadmin/pdf/leitlinien/

GPS_revision2-final_august2014.pdf Letzter Zugriff am 07.03.2016

15 Swart E, Ihle P, Gothe H, Matusiewicz D, Hrsg. Routinedaten im Gesundheitswesen. Handbuch Sekundärdatenanalyse: Grundlagen, Methoden und Perspektiven. 2. vollst. überarb. Auflage. Bern: Huber; 2014

16 Horenkamp-Sonntag D, Linder R, Verheyen F. Analyse bundesweiter GKV-Routinedaten: Zusammenhang zwischen regionalen Morbiditätsveränderungen und Leistungsinanspruchnahmen. 9. Deutscher Kongress für Versorgungsforschung und 5. Jahrestagung Aktionsbündnis Patientensicherheit. Bonn 2010

http://www.tk.de/centaurus/servlet/ contentblob/236372/Datei/2428/

Morbiditaetsveraenderungsanalysen-

Versorgungsforschungskongress-2010-Poster.pdf Letzter Zugriff am 07.03.2016

17 Horenkamp-Sonntag D, Linder R, Wenzel F et al. Datenzugang und Datenvalidierung: Prüfung der Datenqualität und Validität von GKV-Routinedaten. In: Swart E, Swart E, Ihle P et al, Hrsg. Routinedaten im Gesundheitswesen. Handbuch Sekundärdatenanalyse: Grundlagen, Methoden und Perspektiven. 2. vollst. überarb. Auflage. Bern: Huber; 2014: 314-329

18 Chenot J, Haupt C, Gerste B. Zeitliche Trends bei der Versorgung von Rückenschmerzpatienten. In: Klauber ], Günster C, Gerste B et al, Hrsg. Versorgungs-Report 2013/2014. Stuttgart: Schattauer; 2014: 155-184

19 Schäfer T, Pritzkuleit R, Hannemann F et al. Trends und regionale Unterschiede in der Inanspruchnahme von Wirbelsäulenoperationen. In: Klauber ], Geraedts M, Friedrich J, Wasem J, Hrsg. Krankenhaus-Report 2013. Stuttgart: Schattauer; 2013: 111-133

20 Marschall U, L'hoest U, Wolik A. Vergleich der Kosteneffektivität von Operation, multimodaler und interventioneller Schmerztherapie bei Rückenschmerzen: Eine Analyse mit Krankenkassendaten“. In: Repschläger U, Schulte C, Nicole Osterkamp N (eds.): BARMER GEK Gesundheitswe- 
sen aktuell 2012. Berlin: Barmer GEK; 2012:

262-285

http://presse.barmer-gek.de/barmer/web/Portale/ Presseportal/Subportal/Presseinformationen/

Archiv/2012/120829-Rueckenschmerzen/

PDF-Rueckenschmerzen.pdf

Letzter Zugriff am 07.03.2016

21 Linder R, Schneider U, Köthemann, Verheyen F. Ärztliches Verordnungsverhalten von potenziell inadäquaten Medikamenten für Ältere Menschen. Dtsch Med Wochenschr 2014; 139: 983-989

22 Neubauer S, Zeidler J, Schilling T et al. Unter welchen Voraussetzungen eignen sich GKVRoutinedaten zur Überprüfung von Leitlinien im Versorgungsalltag? - Eine Analyse anhand der Indikation Herzinsuffizienz. 58. Jahrestagung der Deutschen Gesellschaft für Medizinische Informatik, Biometrie und Epidemiologie. Lübeck 2013 http://www.egms.de/static/en/meetings/ gmds2013/13gmds226.shtml Letzter Zugriff am 07.03.2016
23 Linder R, Zeidler J, Schilling T et al. Stents hinterfragt: Werden Patienten mit koronaren Herzerkrankungen leitliniengerecht versorgt? 58. Jahrestagung der Deutschen Gesellschaft für Medizinische Informatik, Biometrie und Epidemiologie. Lübeck 2013

http://www.egms.de/static/en/meetings/ gmds2013/13gmds194.shtml Letzter Zugriff am 07.03.2016

24 Schneider U, Linder R, Hagenmeyer E-G et al. Analyse diagnostischer Maßnahmen mit GKVRoutinedaten: Zur Richtlinienkonformität der Stufendiagnostik bei vermuteter Schlafapnoe. 6 . Jahrestagung der Deutschen Gesellschaft für Gesundheitsökonomie. München 2014 http://file.dggoe.de/jahrestagung_2014/ Tagungsprogramm_DGGOE2014_Web_2014-0227.pdf

Letzter Zugriff am 07.03.2016

\section{Interessenkonflikt}

Die Autoren geben an, dass kein Interessenkonflikt besteht.

DOI $10.1055 / \mathrm{s}-0042$

101467

Dtsch Med Wochenschr 2016; 141: e96-e103 (c) Georg Thieme Verlag KG. Stuttgart · New York. ISSN 0012-0472 